\title{
THE LOCAL HILBERT FUNCTION OF A PAIR OF PLANE CURVES ${ }^{1}$
}

\author{
S. C. KOTHARI
}

\begin{abstract}
Let $R$ be the local ring of a pair of plane curves at a point. In this paper it is proved that the Hilbert function of such a ring changes by at most one at each stage, and it is essentially nonincreasing.
\end{abstract}

1. Introduction. Let $\mathcal{O}$ be an equicharacteristic two dimensional regular local ring and $f$ and $g$ be two elements of $\theta$ which generate an ideal $\mathcal{Q}$ primary for the maximal ideal of $\theta$. Let $R, \operatorname{Gr}(\theta)$ and $\operatorname{Gr}(R)$ denote respectively the residue-class ring $\theta / U$ and the graded rings of $\theta$ and $R$. $\mathrm{Gr}^{i}(R)$ denotes the $i$ th homogeneous component of $\operatorname{Gr}(R)$ and $H_{i}(R)$ denotes its vectorspace dimension over $\mathrm{Gr}^{0}(R)$. The function $H_{i}(R)$ is referred to as the Hilbert function of $R$. The orders of $f$ and $g$ are denoted by integers $n$ and $m$ respectively.

The following questions were raised by Professor Abhyankar.

Question 1. Is $\sum_{i=0}^{m+n-1} H_{i}(R)>m n$ when $f$ and $g$ are tangential, i.e., when their initial forms have a common factor?

Question 2. Is $\left|H_{i+1}(R)-H_{i}(R)\right| \leqslant 1$ for all nonnegative integers $i$ ?

These questions are answered in the affirmative and the following theorem is proved.

THEOREM. $0 \leqslant H_{i}(R)-H_{i+1}(R) \leqslant 1$ for $i \geqslant \min (n, m)$.

We will denote by $x, y$ a regular system of parameters in $\theta$ and the residue field of $\theta$ by $k . \operatorname{Gr}(\theta)$ will be identified with the polynomial ring $k[X, Y]$ with $X$ and $Y$ denoting initial forms of $x, y$ respectively. With this identification the initial ideal $\bar{\vartheta}$ of $\mathcal{Q}$ will be a homogeneous ideal in $k[X, Y]$. The $i$ th homogeneous component of $\overline{\mathscr{Q}}$ and its dimension over $k$ are denoted by $\overline{\mathscr{Q}}_{i}$ and $\operatorname{dim}\left(\overline{\mathcal{Q}}_{i}\right)$ respectively. $F$ and $G$ denote initial forms of $f$ and $g$. The definitions of the terms used here can be found in [3].

2. For the purpose of the following lemma $\theta$ will denote the power series ring $k[[x, y]]$ and the field $k$ is assumed to be infinite.

LEMMA. Let $f$ and $g$ be such that $m \geqslant n, g=x g_{1}$ with $g_{1}$ in $\theta$ and $X$ does not divide $F$. Let $\mathcal{V}$ be the ideal generated by $f$ and $g_{1}$ and $\widetilde{\mathcal{V}}$ be its initial ideal. Then $\operatorname{dim}\left(\overline{\mathcal{Q}}_{i+1}\right)=\operatorname{dim}\left(\overline{\mathscr{V}}_{i}\right)+1$ for all $i \geqslant n$.

Received by the editors December 14, 1977.

AMS (MOS) subject classifications (1970). Primary 14H99; Secondary $13 \mathrm{H} 10$.

Key words and phrases. Hilbert function, initial forms, initial ideal.

'This work constitutes a part of the author's Ph.D. thesis at Purdue University. 
Proof. If $W_{1}, W_{2}, \ldots, W_{r}$ is a basis of $\overline{\mathscr{V}}_{i}$ then $X W_{1}, X W_{2}, \ldots, X W_{r}$ and $Y^{i+1-n} F$ are elements in $\overline{\hat{Q}}_{i+1}$ which are linearly independent. Hence it readily follows that $\operatorname{dim}\left(\overline{\mathcal{Q}}_{i+1}\right) \geqslant \operatorname{dim}\left(\overline{\widetilde{V}}_{i}\right)+1$.

Let $t_{1}=y^{i+1-n_{f}}$. We prove that there exist elements $t_{2}, t_{3}, \ldots, t_{s}$ in $\mathscr{Q}$, each divisible by $x$ and such that their initial forms together with $Y^{i+1-n} F$ form a basis of $\bar{Q}_{i+1}$. First choose elements $t_{2}^{*}, t_{3}^{*}, \ldots, t_{s}^{*}$ such that their initial forms and $Y^{i+1-n} F$ form a basis of $\bar{Q}_{i+1}$. If $t_{j}^{*}$ is divisible by $x$ then take $t_{j}=t_{j}^{*}$, otherwise $t_{j}$ is chosen as follows. By Weierstrass preparation theorem,

$$
\begin{aligned}
t_{1} & =\delta\left[y^{i+1}+c_{1}(x) y^{i}+\cdots+c_{i+1}(x)\right] \text { and } \\
t_{j}^{*} & =\eta\left[y^{p}+d_{1}(x) y^{p-1}+\cdots+d_{p}(x)\right],
\end{aligned}
$$

where $\delta$ and $\eta$ are units and $c_{1}(x), \ldots, c_{i+1}(x), d_{1}(x), \ldots, d_{p}(x)$ are power series in $x$ divisible by $x$. Since $m \geqslant n, p$ will be bigger than or equal to $i+1$. Let $t_{j}=t_{j}^{*}-\eta \delta^{-1} y^{p-i-1} t_{1}$. The elements $t_{2}, t_{3}, \ldots, t_{s}$ chosen this way are clearly divisible by $x$ and their initial forms together with $Y^{i+1-n} F$ form a basis of $\overline{\mathcal{Q}}_{i+1}$.

Let $t_{j}=a_{j} f+b_{j} g$ for $j \geqslant 2$. For $j \geqslant 2, t_{j}$ is divisible by $x$, write $t_{j}=x w_{j}$ for $j>2$, also $g=x g_{1}$. We get $x w_{j}=a_{j} f+x b_{j} g_{1}$ which implies that $x$ divides $a_{j}$ and consequently $w_{j}$ belongs to $\mathcal{V}$. The initial forms of $w_{j}$ are in $\overline{\mathcal{V}}_{i}$ and linearly independent and hence $\operatorname{dim}\left(\overline{\mathcal{V}}_{i}\right)+1 \geqslant \operatorname{dim}\left(\overline{\mathscr{Q}}_{i+1}\right)$. This completes the proof of the lemma that $\operatorname{dim}\left(\overline{\widetilde{Q}}_{i+1}\right)=\operatorname{dim}\left(\overline{\mathscr{V}}_{i}\right)+1$ for $i>n$.

3.

Definition. The initial forms of a pair of generators of $\mathcal{Q}$ are said to be irredundant if none of them is a multiple of the other.

Observe that if a pair of generators of $\mathcal{Q}$ is such that their initial forms are irredundant then the sum of the degrees of the initial forms and the degree of the g.c.d. of the initial forms are two well-defined numbers dependent only on the ideal $U$. These numbers will be denoted by $\alpha(U), \beta(U)$ respectively and are used for induction in the proof of the following theorem.

THEOREM. $0 \leqslant H_{i}(R)-H_{i+1}(R) \leqslant 1$ for all $i \geqslant \min (n, m)$.

Proof. The completion $\hat{R}$ of $R$ is isomorphic to $\hat{\theta} / \hat{Q}$, where $\hat{\theta}$ is the completion $\hat{\theta}$ and $\hat{Q}=Q \hat{\theta}$ and also since $R$ and $\hat{R}$ have isomorphic graded rings $H_{i}(R)=H_{i}(\hat{R})$. Hence without loss of generality we assume that the rings $\theta$ and $R$ are complete. By Cohen's structure theorem $\theta$ is then isomorphic to the power series ring $k[[x, y]]$.

Since $\operatorname{Gr}(R) \simeq k[X, Y] / \overline{\mathscr{Q}}$ and $H_{i}(R)=i+1-\operatorname{dim}\left(\overline{\mathcal{Q}}_{i}\right)$, the theorem is equivalent to proving $1 \leqslant \operatorname{dim}\left(\bar{\vartheta}_{i+1}\right)-\operatorname{dim}\left(\bar{\vartheta}_{i}\right) \leqslant 2$ for all $i \geqslant \min (n, m)$. The computation of $\operatorname{dim}\left(\overline{\mathcal{Q}}_{i}\right)$ is fairly straightforward for $i<\min (n, m)+1$ and hence the above inequality easily checked for $i=\min (n, m)$. In view of this it is sufficient to prove $1 \leqslant \operatorname{dim}\left(\bar{\vartheta}_{i+1}\right)-\operatorname{dim}\left(\bar{\vartheta}_{i}\right)<2$ for $i>\min (n$, $m)+1$. We give a proof of this statement assuming that the field $k$ is infinite. 
The proof when $k$ is finite can be reduced to that case by extending $k$ by adjoining an indeterminate.

Let $T_{1}, T_{2}, \ldots, T_{r}$ be a basis over $k$ of $\bar{Q}_{i}$ for $i \geqslant \min (n, m)+1$. If $T_{j}$ is an element of the basis least divisible by $X$ then $Y T_{j}$ and $X T_{1}, X T_{2}, \ldots, X T_{r}$ are linearly independent. These elements are in $\bar{Q}_{i+1}$ and we have that $\operatorname{dim}\left(\overline{\mathcal{Q}}_{i+1}\right)-\operatorname{dim}\left(\overline{\mathcal{Q}}_{i}\right) \geqslant 1$ for $i \geqslant \min (n, m)+1$.

It remains to be proved that $\operatorname{dim}\left(\overline{\mathscr{Q}}_{i+1}\right)-\operatorname{dim}\left(\overline{\mathcal{Q}}_{i}\right) \leqslant 2$ for $i \geqslant \min (n$, $m)+1$. Without loss of generality we can assume that $n \leqslant m$ and that the initial forms of $f$ and $g$ are irredundant. Since the field $k$ is infinite we can, if necessary by making suitable linear transformation in $x$ and $y$, assume that $X$ does not divide the initial form of $f$. Then we show that there exists a $g^{*}$ divisible by $x$ and such that $f$ and $g^{*}$ generate $\mathcal{U}$ and their initial forms are irredundant. If $x$ divides $g$ then there is nothing to prove hence assume that $x$ does not divide $g$. By Weierstrass preparation theorem

$$
\begin{aligned}
& f=\delta\left[y^{n}+c_{1}(x) y^{n-1}+\cdots+c_{n}(x)\right] \text { and } \\
& g=\eta\left[y^{p}+d_{1}(x) y^{p-1}+\cdots+d_{p}(x)\right]
\end{aligned}
$$

where $p$ is some integer bigger than or equal to $m, \delta, \eta$ are units and $c_{1}(x), \ldots, c_{n}(x), d_{1}(x), \ldots, d_{p}(x)$ are power series in $x$ divisible by $x$. Then $g^{*}=g-\eta \delta^{-1} y^{p-n} f$ is as desired. Let $g^{*}=x g_{1}$ and $\mathfrak{V}$ be the ideal generated by $f$ and $g_{1}$. In view of the lemma it is enough to prove that $\operatorname{dim}\left(\mathbb{V}_{i}\right)-$ $\operatorname{dim}\left(\overline{\mathcal{V}}_{i-1}\right) \leqslant 2$ for $i \geqslant n+1$. Since $\mathcal{T}$ is generated by $f$ and $g_{1}, \beta(\mathcal{V}) \leqslant$ $\beta(\mathcal{U})$ and if $\beta(\mathscr{V})=\beta(\mathcal{U})$ then $\alpha(\mathfrak{V})<\alpha(\mathcal{U})$, hence the proof of the theorem follows by induction.

Now in the following corollaries we answer the questions raised by Professor Abhyankar. If $f$ and $g$ are nontangential, i.e., if their initial forms are coprime then $H_{i}(R)$ depends only on the orders of $f$ and $g$, and we will denote it by $H_{i}(n, m)$. It is easily checked that $\sum_{i=0}^{\infty} H_{i}(n, m)=m n$ and $H_{i}(n$, $m)=0$ for all $i \geqslant m+n-1$.

COROLLARY 1. If $f$ and $g$ are tangential then $\sum_{i=0}^{m+n-1} H_{i}(R)>m n$.

Proof. Let $p>0$ be the degree of the g.c.d. of the initial forms of $f$ and $g$. It is easily checked that $H_{i}(R)=H_{i}(n, m)$ for $0 \leqslant i \leqslant m+n-p-1$ and $H_{m+n-p}(R)=H_{m+n-p}(n, m)+1$. By the above theorem, $H_{i}(R)$ decreases by at most one at each stage for $i \geqslant \min (n, m)$ whereas $H_{i}(n, m)$ decreases by one at each stage for $i \geqslant \min (n, m)$ until it becomes zero. It follows that $H_{i}(R) \geqslant H_{i}(n, m)$ for all $i$ and

$$
\sum_{i=0}^{m+n-1} H_{i}(R)>\sum_{i=0}^{m+n-1} H_{i}(n, m)=m n .
$$

COROLlaRY 2. $\left|H_{i+1}(R)-H_{i}(R)\right| \leqslant 1$ for all nonnegative integers.

Proof. For $0 \leqslant i \leqslant \min (n, m)$ this is checked by direct calculation and for $i>\min (n, m)$ this follows from the theorem. 
We deduce the following result of Bertini cited in [1].

COROLlaRY 3. Let $\alpha$ be the intersection multiplicity of $f$ and $g$ and $e$ be the largest integer such that $H_{i}(R)>0$ for all $i \leqslant e$ then $e<\alpha+m+n-m n-$ 2.

Proof. Since, as observed in the proof of Corollary $1, H_{i}(R) \geqslant H_{i}(n, m)$ for all $i$ and $H_{i}(n, m)=0$ for $i \geqslant m+n-1$ hence $\sum_{i=0}^{e} H_{i}(R) \geqslant$ $\sum_{i=0}^{m+n-2} H_{i}(n, m)+(e-m-n+2)$. But $\sum_{i=0}^{e} H_{i}(R)=\alpha$, the intersection multiplicity and $\sum_{i=0}^{m+n-2} H_{i}(n, m)=m n$ hence the result follows.

\section{REFERENCES}

1. L. Berzolari, Allgemeine Theorie der Hoheren Ebenen Algebraischen Kurven, Encyklopadie der Mathematischen Wissenschaften IIIC4, 1932.

2. M. Nagata, Local rings, Interscience, New York, 1962.

3. O. Zariski and P. Samuel, Commutative algebra, Vol. II, Van Nostrand, Princeton, N. J., 1962.

Department of Mathematics, SOUTHERn Illinois University, Carbondale, Illinois 62901 